\title{
Investigation of a cross-correlation based optical strain measurement technique for detecting radial growth on a rotating disk
}

\author{
Michelle M. Clem and Mark R. Woike \\ National Aeronautics and Space Administration \\ Glenn Research Center \\ Cleveland, Ohio 44135 \\ Ali Abdul-Aziz \\ Cleveland State University \\ Cleveland, Ohio 44115
}

\begin{abstract}
The Aeronautical Sciences Project under NASA's Fundamental Aeronautics Program is extremely interested in the development of novel measurement technologies, such as optical surface measurements in the internal parts of a flow path, for in situ health monitoring of gas turbine engines. In situ health monitoring has the potential to detect flaws, i.e. cracks in key components, such as engine turbine disks, before the flaws lead to catastrophic failure. In the present study, a cross-correlation imaging technique is investigated in a proof-of-concept study as a possible optical technique to measure the radial growth and strain field on an already cracked sub-scale turbine engine disk under loaded conditions in the NASA Glenn Research Center's High Precision Rotordynamics Laboratory. The optical strain measurement technique under investigation offers potential fault detection using an applied high-contrast random speckle pattern and imaging the pattern under unloaded and loaded conditions with a CCD camera. Spinning the cracked disk at high speeds induces an external load, resulting in a radial growth of the disk of approximately $50.0-\mu \mathrm{m}$ in the flawed region and hence, a localized strain field. When imaging the cracked disk under static conditions, the disk will be undistorted; however, during rotation the cracked region will grow radially, thus causing the applied particle pattern to be 'shifted'. The resulting particle displacements between the two images will then be measured using the two-dimensional cross-correlation algorithms implemented in standard Particle Image Velocimetry (PIV) software to track the disk growth, which facilitates calculation of the localized strain field. In order to develop and validate this optical strain measurement technique an initial proof-of-concept experiment is carried out in a controlled environment. Using PIV optimization principles and guidelines, three potential speckle patterns, for future use on the rotating disk, are developed and investigated in the controlled experiment. A range of known shifts are induced on the patterns; reference and data images are acquired before and after the induced shift, respectively, and the images are processed using the crosscorrelation algorithms in order to determine the particle displacements. The effectiveness of each pattern at resolving the known shift is evaluated and discussed in order to choose the most suitable pattern to be implemented onto a rotating disk in the Rotordynamics Lab. Although testing on the rotating disk has not yet been performed, the driving principles behind the development of the present optical technique are based upon critical aspects of the future experiment, such as the amount of expected radial growth, disk analysis, and experimental design and are therefore addressed in the paper.
\end{abstract}

\section{MOTIVATION AND INTRODUCTION}

Gas turbine engines operate in severe environmental conditions and are therefore repeatedly exposed to high thermal and mechanical loads. The cumulative effects of these external forces lead to high stresses and strains on the engine components, such as the engine turbine disks. Strain on an engine turbine disk is a precursor to a fault, such as a crack forming in the disk, which may eventually lead to a catastrophic failure. Current gas turbine engine fault detection techniques are limited to periodic physical inspections and schedule-based maintenance of engine components to ensure 
their integrity over the lifetime of the engine. Unfortunately, these methods have their limitations and failures have been experienced, leading to engine shutdowns and unplanned maintenance. Typically, strain is detected and measured using strain gages, which are a mature and reliable physical measurement technique, however they are intrusive and are therefore not easily implemented on an engine turbine disk, where transmitting signals to and from the sensor become troublesome. In addition, strain gages only provide point measurements, requiring numerous gage installations and interpolation in between points in order to obtain a global strain field. There are currently no in situ measurement techniques available to non-intrusively measure the strains experienced in the turbine engine and on its rotating components, including the turbine disk. Therefore, in an effort to prevent future failures caused by undetected strains and faults, the development of fault detection techniques in the form of new sensor technologies or methods for the in situ structural health monitoring of gas turbine engines and their components is of high interest to NASA. Currently, external sensors are under development at NASA Glenn Research Center's (GRC) High Precision Rotordynamics Laboratory including Microwave Blade Tip Clearance (MBTC) ${ }^{1-3}$ sensors and Capacitive Blade Tip Clearance (CBTC) sensors ${ }^{4}$. Although these techniques would be non-intrusive to the turbine engine disks, they still require hard mounting through the engine casing. Alternatively, optical strain measurement techniques, such as using a Moiré Pattern ${ }^{5}$, are also being investigated due to their non-invasive and often less complex setup. The optical technique under investigation in the present study is being pursued by the Innovative Measurements Task within the Aeronautical Sciences Project under NASA's Fundamental Aeronautics Program, to develop optical surface measurements for the internal parts of a flow, such as the engine turbine disk. The present optical strain measurement technique under investigation offers potential fault detection by measuring the radial growth, which enables the calculation of strain, of an already cracked sub-scale engine turbine disk, while operating under loaded conditions; however the technique is first being studied in a controlled proof-of-concept experiment, in order to validate and better understand the limitations of the technique.

It is envisioned to implement this optical strain measurement technique to measure the radial growth resulting from a load applied to an already cracked sub-scale turbine engine disk in NASA GRC's High Precision Rotordynamics Laboratory. Knowing the radial growth of the disk allows for potential strain field mapping. For this future investigation, a notch will be machined on to the disk to simulate a crack. Two data images will be acquired: a reference image of the cracked disk will be acquired under static conditions at $0 \mathrm{rpm}$ (unloaded condition) while a secondary image will be acquired of the same disk at $15000 \mathrm{rpm}$ (loaded condition). Spinning the cracked disk at high speeds induces an external load, resulting in a radial growth of the disk in the flawed region and hence, a localized strain field. When imaging the cracked disk under static conditions, the disk will appear undistorted. However, during rotation the cracked region will grow radially, thus causing the applied particle pattern to appear 'shifted'. The two images will then be processed using the cross-correlation algorithms implemented in standard Particle Image Velocimetry (PIV) software to track the disk growth. The radial growth, of this newly fabricated aluminum disk, experienced under high revolutions has yet to be verified with experimental measurements, such as the MBTC sensors. Therefore, as a first step and the focus of this paper, it is necessary to perform a proof-of-concept experiment in order to develop and validate the optical technique in a controlled environment where a known shift of the pattern can be induced into the system. When conducting the investigation in a controlled environment, any resulting error can be more easily diagnosed and improved upon without the uncertainty and complications of a rotating disk. In addition, several pattern designs are investigated including adhesive and paint, both of which cannot be easily removed or may cause permanent damage to the disk. The difficulty of removing the tested patterns and the high cost of potential replacement disks are additional reasons to perform a proof-of-concept experiment involving lesser expensive thin plates of aluminum.

Using PIV optimization principles and guidelines, three potential patterns are developed consisting of high-contrast random particles, including retro reflective adhesive, retro-reflective "paint", and flat black speckled paint. The patterns are applied to thin aluminum plates, which are mounted onto a translation mechanism with a fine micrometer and are translated a known amount perpendicular to the optical axis, thus inducing a known shift. Reference and data images are acquired of each pattern before and after the known pattern displacement, respectively. The image pair is subsequently processed using the cross-correlation algorithms. A series of three known displacements ranging from the maximum expected radial growth of the disk $(50.0-\mu \mathrm{m})$ to the micrometer's smallest translational increment $(10-\mu \mathrm{m})$ are induced into the system for each pattern. The effectiveness of each pattern at resolving the displacements is evaluated and discussed in order to select the most suitable pattern to be implemented on the rotating disk in the Rotordynamics Lab. Future development and refinement of the technique may lead to the ability of providing global strain and growth mapping of an object in laboratory strain diagnostic work for both static and rotating objects due to its ease of use and minimal setup. In addition, once the technique has been validated for use on a rotating disk, the physical application of the technique can then be further refined for potential in situ health monitoring of engine turbine disks. 


\section{THEORY}

The cross-correlation algorithms implemented in PIV software are used to calculate image displacements between particles entrained in a flow moving a finite distance between two closely spaced images in time to calculate flow velocities. In the case of the present study, the cross-correlation algorithms are used to calculate the particle pattern displacements between the reference image of the static disk and the image of the rotating disk under loaded conditions. When imaging the cracked disk under static conditions, the disk will appear un-distorted. However, during rotation the cracked region will grow radially, thus causing the applied pattern to appear 'shifted', resulting in a pattern (image) displacement. The pattern applied to the disk consists of a high-contrast random particle pattern to imitate a typical PIV image. The apparent shift of the pattern, due to radial growth of the cracked disk under loaded conditions, makes the particles appear as if they traveled a finite distance between the two images, therefore making cross-correlation based PIV algorithms a good choice to calculate the particle displacement. Due to the fact that PIV algorithms are used to determine the resulting image displacements, the optimization principles of PIV drive the design of the particle patterns investigated in the proof-of-concept experiment. In addition, the density of the particles in a typical PIV image is such that the particle pattern is considered to be speckle' in which the particle images overlap and the resultant image is a random interference pattern. The goal for the high-contrast random patterns being investigated in the current study for use on the rotating disk is to create an optimized speckle pattern that appears as a "look a-like" PIV image when imaged through the optical system.

Particle Image Velocimetry is a technique for measuring the in-plane velocity field of a flow seeded with tracer particles. A pulsed laser sheet is used to illuminate the tracer particles entrained in the flow and the light scattering off of the particles is collected normal to the laser light sheet and is imaged onto a CCD detector. The positions of the particles are captured onto the detector for two images closely spaced in time. The flow velocity, $v$, can be inferred from the particle displacement between the two images using Eq. 1, where $\Delta x$ is the particle displacement, $\Delta t$ is the time interval between images/light sheet pulses, and $M$ is the magnification of the imaging system.

$$
v=\frac{\Delta x}{M(\Delta t)}
$$

A schematic of a typical PIV system is shown in Fig. 1(a). It can be seen in the schematic that a light sheet of thickness, $\Delta Z_{o}$, illuminates the particles entrained in the flow and an imaging lens placed at distance, $Z_{o}$, away images the particle positions onto the image plane at distance, $z_{o}$, away. The particle displacement from the two successive images is processed by dividing the image up into small interrogation windows (sub-regions) and cross-correlating the corresponding sub-regions. The sub-region from image one is cross-correlated via FFT operations with the same subregion from image two. The location of the displacement peak from the origin on the correlation plane gives the velocity vector magnitude and direction. This process is illustrated in Fig. 1(b) (acquired from [6]) and is repeated over the entire image plane resulting in spatially averaged displacement vectors.
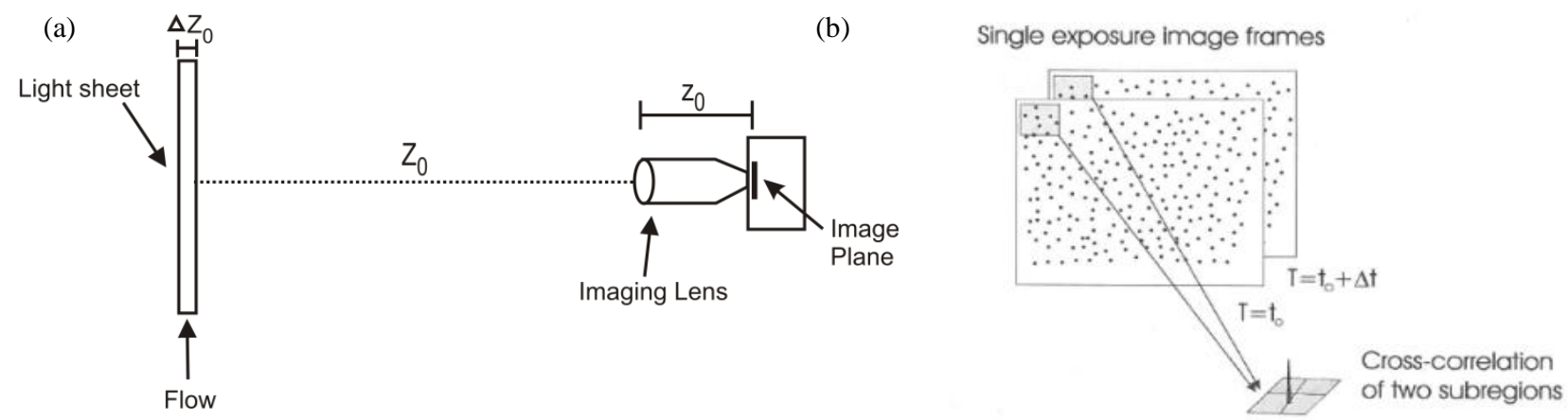

Figure 1. (a) Schematic of a typical PIV setup and (b) illustration of the sub-region cross-correlation process that is repeated over the entire image resulting in spatially averaged displacement vectors [6].

The minimum error in the correlation peak position estimate occurs when the diameter of the imaged particle is approximately 1- 2 pixels. When imaging the random particle pattern, the diffracted blur spot of the particles, instead of the actual particles, gets imaged onto the detector. The diffraction limited blur circle diameter, $d_{d i f f}$, is derived from the 
Airy Function and is shown in Eq. 2, where $M$ is the optical system magnification, $\lambda$ is the wavelength of light and $f \#$ is the $\mathrm{f} /$ number setting of the camera lens. This diffraction limit is also known as the point spread function (PSF) of the lens in the optical system. Equation 3 shows that the PSF and the physical particle diameter, $d_{p}$, multiplied by the system magnification are added in quadrature to determine the effective particle diameter, i.e. the imaged particle size on the detector, $d_{e}{ }^{7}$.

$$
\begin{gathered}
d_{\text {diff }}=2.44(1+M) \lambda f \# \\
d_{e}=\sqrt{\left(d_{p} M\right)^{2}+(2.44(1+M) \lambda f \#)^{2}}
\end{gathered}
$$

Successful cross-correlation of the sub-regions across the entire image depends on the ability to identify the displacement correlation peak, with respect to the random correlation noise. The correlation peak signal to noise ratio is proportional to the number of particles in the sub-region; the optimal number of particles per sub-region is nominally 10. The accuracy of the PIV displacement estimates is primarily determined by the ratio of the correlation peak size, $d$, to the sub-region size $N$, shown in Eq. 4. The correlation peak size is proportional to $\sqrt{2} d_{e}$, where $d_{e}$ is the effective particle diameter. Furthermore, the maximum displacement in the correlation plane is limited by the sub-region size. In order to avoid aliasing in the correlation plane, the displacement should be less than $1 / 4^{\text {th }}$ of the sub-region size. By using interpolation techniques, the correlation peak position error can be determined to sub-pixel accuracy. Nominal values for the peak position error, i.e. $\sigma_{\Delta x}$, are on the order of 0.1-pixel for particles spanning 1-2 pixels ${ }^{8-11}$.

$$
\sigma_{\Delta x}=\frac{d}{N}=\frac{\sqrt{2} d_{e}}{N}
$$

As stated previously, the optimization guidelines of PIV drive the design of the potential patterns being investigated in the present proof-of-concept experiment. Images are acquired of the patterns before and after the patterns are displaced a known amount. Cross-correlation of the images using PIV software is performed in order to calculate the image displacements. In summary, the three optimization principles being used in the development of the high-contrast random particle pattern are:

1. The imaged particle (effective particle diameter) spans 1-2 pixels

2. There are nominally 10 particles per sub-region

3. The sub-region is chosen, such that maximum displacement is less than $1 / 4^{\text {th }}$ of the sub-region size

A more thorough and detailed review of the fundamentals and optimization principles of Digital Particle Image Velocimetry (DPIV) can be found in [11-12].

\section{EXPERIMENTAL SETUP AND PROCEDURE}

\section{A. Proof-of-concept experiment for the optical technique development and validation}

A proof-of-concept experiment is designed in order to develop and validate the optical strain measurement technique under investigation. A top-view of the bench top experiment shown in Fig. 5 replicates the proposed design that will be implemented in the Rotordynamics Lab. A 5W, 505-nm LED is used as the incident light source for the system, which uniformly illuminates a plate with a high contrast random particle pattern adhered onto its front side. The plate is mounted onto a translation mechanism equipped with a fine micrometer in order to shift the pattern a known amount perpendicular to optical axis. Taking into consideration the $50.8-\mathrm{mm}$ crack to be machined into the aluminum disk and the resulting expected radial growth of approximately 50- $\mu \mathrm{m}\left(2\right.$-mils), it was decided to make the imaging area, $y_{o}$, approximately 74-mm to ensure sufficient coverage of the deformed/strained area surrounding the crack. A Princeton Instruments EC11000 scientific-grade CCD camera, with a detector size of 36-mm (H) x 24-mm (V) consisting of 4008 pixels x 2672 pixels equipped with a DC-Nikkor 135-mm, f/2D lens is used to image the applied pattern. In order to optimize the resulting image's spatial resolution, the strained area of the disk was imaged onto the horizontal side of the detector $\left(y_{i}=36-\mathrm{mm}\right)$ and the camera or object distance, $x_{o}$, was set to approximately $421-\mathrm{mm}$ due to facility constraints. 
It is important to note, that camera lenses have a minimum focus distance, where the lens is unable to focus on anything shorter than this distance. In the present experiment the Nikkor $135-\mathrm{mm}$ focal length lens has a minimum focus distance of $1.1-\mathrm{m}$, which is greater than the object distance of $421-\mathrm{mm}$. Therefore, an extension tube was placed between the camera lens and the detector in order to increase the imaging distance, thus changing the effective focus of the lens. A Nikon extension tube was not readily available, so an F-mount adaptor and two 12.7-mm lens tubes were used to create an extension tube and is shown in Fig. 6. The resulting horizontal field-of-view is $73.25-\mathrm{mm}$ giving a scale factor of 54.72 pixels $/ \mathrm{mm}$.

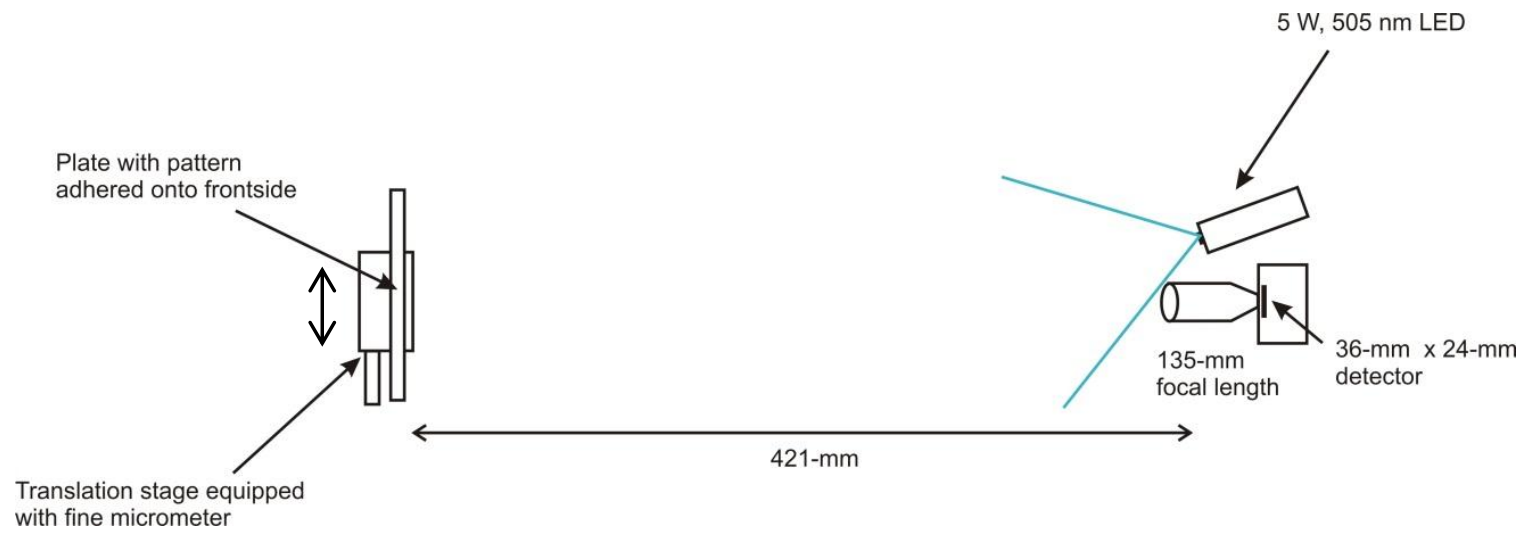

Figure 5. A top-view schematic of the bench top proof of concept experiment to develop and validate the optical strain measurement technique under investigation. The micrometer adjustment was used to translate the pattern perpendicular to the optical axis.

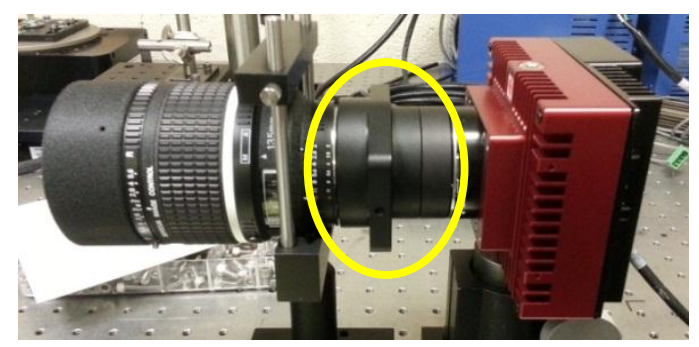

Figure 6. Extension tube used to increase the image distance to change the minimum focus of the lens.

Three different particle patterns consisting of micro glass beads, retro-reflective adhesive and flat black paint were applied to thin aluminum plates and evaluated using the PIV optimization guidelines. The glass beads, reflective adhesive, and black speckled patterns, as imaged through the optical system, are shown in Figs 7(a)-7(c), respectively. The imaged area for each pattern is $73.25-\mathrm{mm}$ (horizontal) by $48.83-\mathrm{mm}$ (vertical). The glass beads (size to be discussed in Section IV) were adhered to the aluminum plate using spray adhesive. The adhesive was used to coat the aluminum plate and the glass beads were sprinkled onto the wet plate by hand as uniformly as possible. Once a layer of glass beads was applied, the plate was picked up and tapped onto the table in order to "knock off" any excess or loose beads. The plate was also gently shaken in an attempt to avoid the glass beads lumping together. The process was repeated until the plate appeared to be covered in a uniform and relatively high density of glass beads. Unfortunately, the density and uniformity of the glass beads were hard to control, resulting in non-uniform areas, some of which were too dense and appearing more as a "clumpy" white powder than the desired crystal-like appearance. An alternative approach to using the glass beads was to investigate a $3 \mathrm{M}$ retro-reflective adhesive. The reflective adhesive contains micro-prisms (size not specified by manufacturer) that are bonded to a flexible, glossy, UV-stabilized polymeric film, which was then cut and applied to the aluminum plate. The reflective adhesive is an undesirable pattern material to use for application onto the rotating disk due to the complexity of verifying that the adhesive would grow in unison and the same amount as the loaded disk. However, the motive behind evaluating the adhesive was to investigate a pattern that was guaranteed to have a uniform application and density of reflective particles to compare to the non-uniform glass beads. Lastly, flat black spray paint was used to create a speckle pattern on the aluminum plate. The spray can was held 
approximately 2 -ft above the plate and was sprayed slightly beyond the plate such that the majority of the paint was coating a piece of scrap paper and only small dots fell and randomly covered the aluminum plate. This was continued until the desired speckle density accumulated onto the plate. Not only was the uniformity and density hard to control, but the speckle size was difficult to control as well. As expected, Figs. 7(a) and 7(b) show that both the retro-reflective glass beads and the reflective adhesive reflect much of the incoming light back on itself toward the source, whereas Fig. 7(c) shows the black speckled pattern does not reflect much of the light. In addition, there is also a noticeable glare spot in the image of the black speckled paint that is dependent on the position of the source and the observer. This glare is disadvantageous due to the possibility of saturation or inaccurate signal to noise ratio and difficulty in achieving uniform illumination over the area of interest. Regarding particle density and particle size, it is obvious that the reflective adhesive, Fig. 7(b), shows the most uniform distribution and size of particles, however it is also evident pockets of air are trapped underneath the adhesive causing raised portions of the pattern. The raised pattern areas may appear in slightly less focus and may also "deflate" over time and may contribute to error in the correlation processing. Both the patterns consisting of the glass beads and black speckled paint applied by hand, Figs. 7(a) and 7(c) respectively, have a less uniform particle density and have large particle sizes as compared to the reflective adhesive. The non-uniform density of the glass beads can be seen by the "cloudiness and clumpiness" nature of the pattern, whereas the non-uniform density of the particles in the black speckled pattern are seen by the dark areas of the pattern consisting of more overlapping speckles than other areas of the plate.

(a)

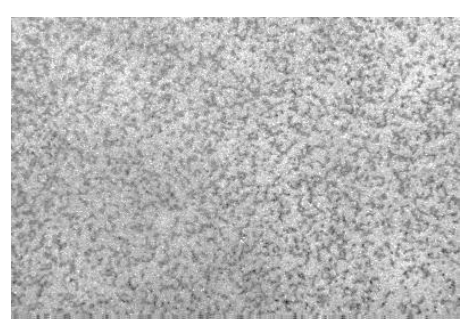

(b)

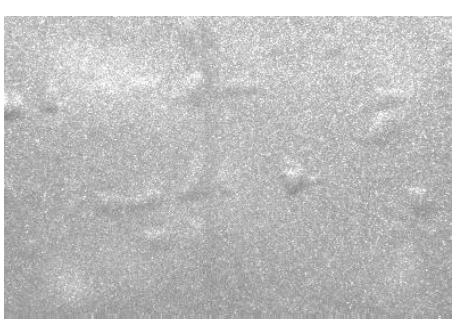

(c)

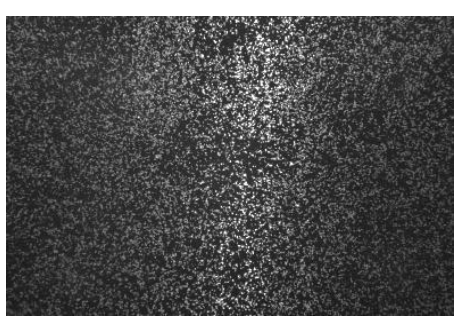

Figure 7. Images depicting the (a) glass beads, (b) retro-reflective adhesive, and (c) speckled flat black paint patterns evaluated using the PIV optimization principles as imaged through the optical system in the proof-of-concept experiment. The imaged area of each pattern is $73.25-\mathrm{mm}$ (horizontal) by 48.83 -mm (vertical).

It is imperative that the pattern particles are in good focus since the optical strain measurement relies on detecting a shift in the particle images. It is predicted that the currently proposed aluminum disk to be used in the Rotordynamics Lab will grow a maximum amount of approximately $50.0-\mu \mathrm{m}$ in the radial direction when spinning at $15,000 \mathrm{rpm}$. The expectation is that the largest amount of growth will occur at the outer edge of the disk, however it is suspected that a much smaller growth will occur immediately surrounding the cracked region. Therefore, it is necessary to investigate the resolvable displacement range that each pattern is capable of detecting. In order to do so, each pattern was mounted onto a translation stage equipped with a fine micrometer (as shown in Fig. 5) which was used to translate the plate a specified amount perpendicular to the optical axis, thus shifting the pattern. Reference and data images were acquired of each pattern before and after the pattern displacements, respectively. The images were subsequently processed using the cross-correlation algorithms available in PIV software. This procedure was carried out 5 times for each pattern, for three known shifts of $50.0-\mu \mathrm{m}, 20.0-\mu \mathrm{m}$, and $10.0-\mu \mathrm{m}$. Although it is possible for the localized strain region surrounding the crack to consist of radial displacements smaller than $10-\mu \mathrm{m}$, this was the smallest increment of the micrometer, hence the smallest known shift capable of being produced.

\section{B. Implementation of the optical technique into the High Precision Rotordynamics Laboratory}

After the optical strain measurement technique has been validated in the present proof-of-concept experiment and the most effective pattern has been chosen, the near term goal is to apply the pattern onto the rotating disk to be tested in the High Precision Rotordynamics Laboratory at NASA GRC in order to detect the radial growth of the cracked disk under loaded conditions. Currently, it is proposed to use a 12.7-mm thick, 254-mm, diameter, 32 blade aluminum disk (shown in Fig. 8) that is expected to grow a maximum amount of approximately $50.0-\mu \mathrm{m}$ in the radial direction when spinning at $15,000 \mathrm{rpm}$. As stated previously, a notch $50.8-\mathrm{mm}$ in length will be machined into the disk in order to simulate a crack/fault. When the disk is spun at $15,000 \mathrm{rpm}$, an external force is induced onto the disk creating a localized strain 
field around the crack and resulting in radial growth. A sample image of the crack to be induced onto the disk is shown in Fig. 9 (the crack shown is from a different disk because the notch in the proposed aluminum disk has not yet been machined). Note the expected radial growth of the disk due to the induced crack is in addition to any other growth that the disk may experience, such as thermal growth. The growth measured using the present optical technique will be compared to reference data acquired from a non-cracked disk operating at the same test conditions. Thus the difference in radial growth measured between the flawed and non-flawed disk operating under loaded conditions, will be taken to be the radial growth of the disk due to the resulting strain field of the induced crack.

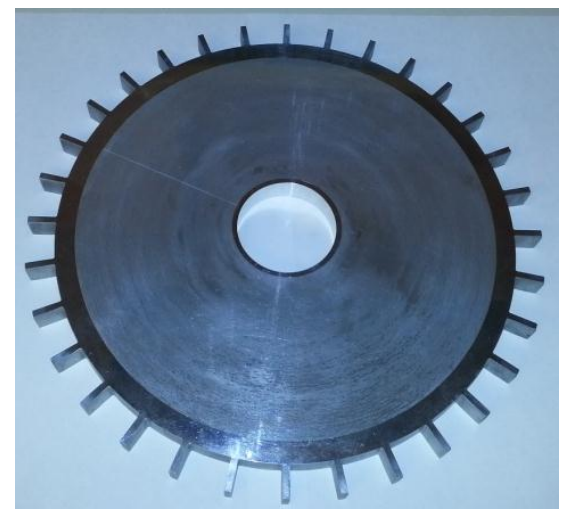

Figure 8. The 12.7-mm thick, 254-mm diameter aluminum disk that will be tested in the Rotordynamics Lab.

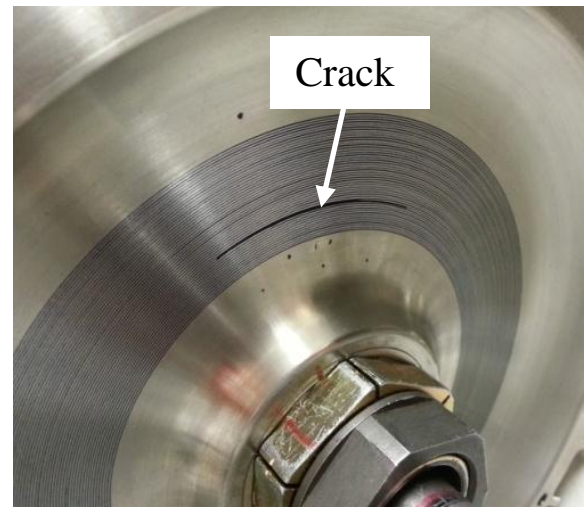

Figure 9. Sample of a simulated crack $50.8-\mathrm{mm}$ in length machined into a disk.

Figure 10 shows NASA GRC's High Precision Rotordynamics Lab, which can accommodate simulated engine rotor disks of up to 254-mm in diameter. The rig has a stainless steel shaft with a length of 781-mm and diameter of 20-mm, which is supported by precision contact ball bearings on each end and has adjustable dampers. An encoder is mounted on the end of the shaft which is used by the control system to provide closed-loop control of rig speed and a secondary optical tachometer is used to record the speed into the data system and to synchronize the data to the rig's rotation. A 12hp custom-built, direct-current $(\mathrm{dc})$ motor is used to rotate the spin rig and the simulated engine disks up to speeds of $15,000 \mathrm{rpm}$

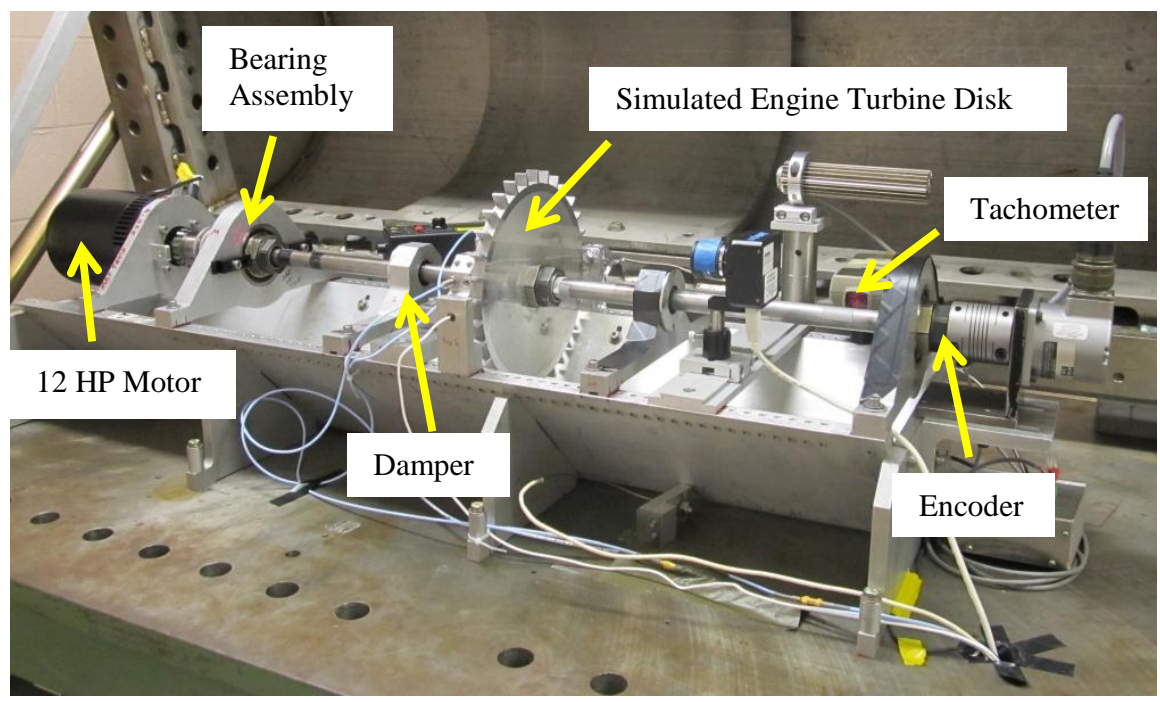

Figure 10. The High Precision Rotorydynamics Lab at NASA GRC in which the most effective pattern will be applied onto a rotating disk. 


\section{RESULTS AND DISCUSSION}

\section{A. Proof-of-concept experiment for optical technique development and validation}

A proof-of-concept experiment is performed to develop and validate the present optical strain measurement technique. The effectiveness of the three potential particle patterns are analyzed using the PIV optimization guidelines previously discussed: the particle displacement should be less than $1 / 4^{\text {th }}$ of the sub-region size, the particle diameter should span approximately 1-2 pixels, and there should be nominally 10 particles per sub-region. Following the guideline that the effective particle diameter, $d_{e}$, should span 1 - 2 pixels, i.e. $18.3-\mu \mathrm{m}-36.5-\mu \mathrm{m}$ using the scale factor, the actual particle diameter, $d_{p}$, necessary for the optimization of the pattern design was calculated using Eq. 3, where $\lambda=505-\mathrm{nm}, f \#=16$, and $M=0.49$ (acquired from the optical setup where $d_{i}=36-\mathrm{mm}$ and $d_{o}=73.25-\mathrm{mm}$ ). In order for $d_{e}$ to not exceed $36.5-\mu \mathrm{m}$ ( 2 pixels), $d_{p}$ must be less than approximately $45-\mu \mathrm{m}$. It is important to note that the smallest achievable $d_{e}$ is limited by the size of the diffraction limited blur circle, given by Eq. 2, which is $29.4-\mu \mathrm{m}$ in the present study. Therefore, the smallest $d_{e}$ capable of being imaged by the current optical system will span approximately 1.6 pixels. To accommodate the small particle sizes necessary for the optimization $\left(d_{p}<45-\mu \mathrm{m}\right)$ it was thought to use retroreflective paint due to its consistency of small glass spheres (on the order of microns) in the paint, which are used to reflect the incoming light rays back onto themselves. Unfortunately, it was found out that $3 \mathrm{M}$ no longer sells its retroreflective paint with the beads already mixed into the paint; the glass beads needed to be purchased separately and applied with some type of bonding agent. After some research, glass beads were purchased from Potters Industries in the closest available size range of $40-\mu \mathrm{m}-100-\mu \mathrm{m}$. Substituting in these values for $d_{p}$ results in a new $d_{e}$ range of 35.3$\mu \mathrm{m}-57.1-\mu \mathrm{m}$ or approximately $2-3$ pixels, which is still an acceptable diameter range ${ }^{9}$. The effective particle diameter could not be calculated for the reflective adhesive since the particles, consisting of micro-prisms, were not specified by the manufacturer. A reliable effective particle diameter could also not be calculated for the black paint speckles due to the inability to control the physical particle sizes.

In order to avoid aliasing in the correlation plane, the particle displacement should be less than $1 / 4^{\text {th }}$ of the sub-region size. It is known from prior computational analysis that the largest expected radial shift to occur on the proposed cracked disk, and therefore the pattern when applied to the disk, is approximately 50.0- $\mu \mathrm{m}$ at $15,000 \mathrm{rpm}$. Using the scale factor, a shift of 50.0- $\mu \mathrm{m}$ corresponds to a pixel shift in the image plane of approximately 2.73 pixels. Therefore, the sub-region size in the present study was set to 16 x16 pixels, thus meeting the PIV optimization criteria. Note a typical PIV sub-region size is on the order of $2^{n}$, such as 32 × 32 pixels, however, spatial resolution increases as the subregion size decreases, which is why a smaller sub-region was chosen that still meets the guidelines. Figs. 11(a)-11(c) show a representative $16 \times 16$ pixel sub-region, depicted by the yellow square, from each of the tested patterns: 11(a) glass beads, 11(b) retro-reflective adhesive, and 11(c) black speckles. Note the sub-regions for the glass beads and the black speckles vary more across the image than the reflective adhesive due to their lesser controlled particle density via the manual application. Therefore, Figs. 11(a) glass beads and 11(c) black speckles depict "average" sub-regions consisting of a region that is neither the densest nor the least dense. It is verified in the image of the $40-\mu \mathrm{m}-100-\mu \mathrm{m}$ glass beads (Fig. 11(a)) that their effective diameter when imaged through the optical system spans $2-3$ pixels as predicted by the calculations. However, only 4 full and 2 partial particles appear in one sub-region instead of the optimal number of nominally 10 particles per sub-region to ensure the proper correlation peak signal to noise ratio. However, it is important to note that having a lesser or greater number of particles does not decrease or increase the accuracy of the correlation peak position. Instead having the optimal number of particles per sub-region increases the probability that the correlation peak of the two sub-regions will be larger than the random noise in the spatial correlation, therefore increasing the likelihood of correctly identifying the correlation peak. Figure 11(b) shows that the reflective adhesive does in fact contain nearly the optimal number of particles per sub-region, however it is also observed that some of the reflective particles appear to span 2 pixels, whereas other particles in the sub-region overlap creating larger combined effective particle diameters of approximately 3-5 pixels. Conversely, Fig. 11(c) shows white "particles" created by the non-overlapping areas of the black speckled paint. It can be seen that the particles tend to lump together and appear less like distinct individual particles like those created with the glass beads shown in Fig. 11(a). The effective particle diameter for the white regions, created by the gaps in the black speckled paint, range from approximately 1-5 pixels. Again, the number of particles occurring in the sample sub-region size is less than the optimal number. 
(a)

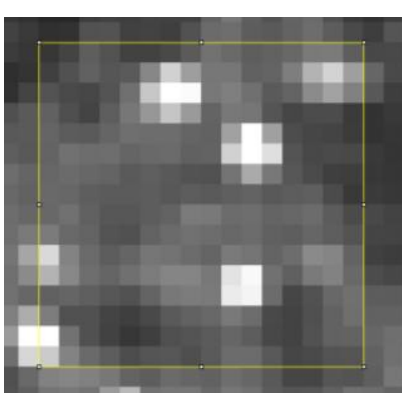

(b)

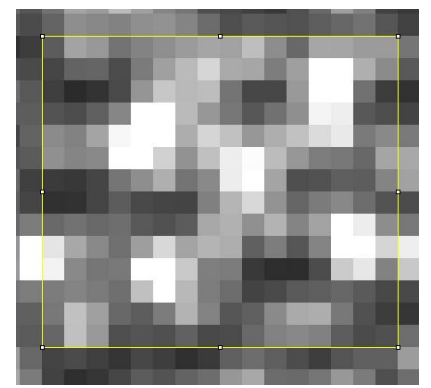

(c)

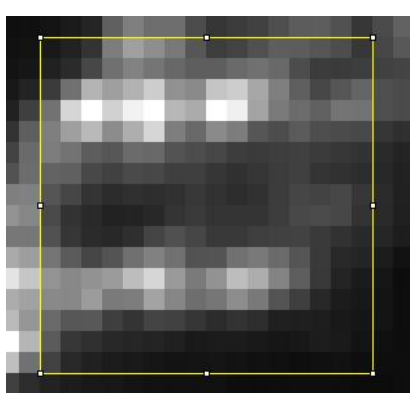

Figure 11. A zoomed in view of a representative16 x 16 pixel sub-region window showing the particle density and the effective particle diameter size (in pixels) for (a) the $40-\mu \mathrm{m}-100-\mu \mathrm{m}$ glass beads, (b) the $3 \mathrm{M}$ retro-reflective adhesive, and (c) the flat black speckled paint.

As stated previously, the expectation is that the largest amount of growth will occur at the outer edge of the flawed disk when spinning at 15,000 rpm, however it is suspected that a much smaller growth will occur immediately surrounding the cracked region. Therefore, it is necessary to investigate the resolvable displacement range that each particle pattern is capable of detecting. In order to do so, each pattern was manually shifted 50.0- $\mu \mathrm{m}, 20.0-\mu \mathrm{m}$, and $10.0-\mu \mathrm{m}$ in the horizontal direction (perpendicular to the optical axis) using the micrometer and setup shown in Fig. 5. Note the patterns were not shifted in the vertical direction; therefore the known vertical shift is $0.0-\mu \mathrm{m}$. A total of 5 reference and data image pairs for each pattern material were processed using a 16x16 pixel sub-region that was translated across each of the entire images to produce spatially averaged displacement vectors. Since the patterns were shifted as a whole in the horizontal direction, ideally every point on the pattern moved the same distance. Therefore, a total of 828,340 spatially averaged displacement vectors resulting from the cross-correlation routine for all 5 image pairs were averaged to produce an overall particle displacement vector, referred to as the "detected shift". Table 1 summarizes the detected shifts and their standard deviation for each particle pattern as compared to the known shifts for both the horizontal and vertical directions.

Table 1: Summary of the detected shifts for each particle pattern compared to the known shift in both the horizontal and vertical directions.

\begin{tabular}{|c|c|c|c|c|c|c|c|}
\hline $\begin{array}{c}\text { Known } \\
\text { Horizontal } \\
\text { Shift } \\
(\mu \mathrm{m})\end{array}$ & $\begin{array}{c}\text { Reflective } \\
\text { Adhesive } \\
\text { Detected } \\
\text { Horizontal } \\
\text { Shift } \\
(\mu \mathrm{m}) \\
\end{array}$ & $\begin{array}{c}40-100 \mu \mathrm{m} \\
\text { Glass Beads } \\
\text { Detected } \\
\text { Horizontal } \\
\text { Shift } \\
(\mu \mathrm{m}) \\
\end{array}$ & $\begin{array}{c}\text { Black Paint } \\
\text { Speckles } \\
\text { Detected } \\
\text { Horizontal } \\
\text { Shift } \\
(\mu \mathrm{m}) \\
\end{array}$ & $\begin{array}{c}\text { Known } \\
\text { Vertical } \\
\text { Shift } \\
(\mu \mathrm{m})\end{array}$ & $\begin{array}{c}\text { Reflective } \\
\text { Adhesive } \\
\text { Detected } \\
\text { Vertical } \\
\text { Shift } \\
(\mu \mathrm{m}) \\
\end{array}$ & $\begin{array}{c}40-100 \mu \mathrm{m} \\
\text { Glass Beads } \\
\text { Detected } \\
\text { Vertical } \\
\text { Shift } \\
(\mu \mathrm{m}) \\
\end{array}$ & $\begin{array}{c}\text { Black Paint } \\
\text { Speckles } \\
\text { Detected } \\
\text { Vertical } \\
\text { Shift } \\
(\mu \mathrm{m}) \\
\end{array}$ \\
\hline & $46.86 \pm 3.01$ & $46.56 \pm 2.47$ & $43.14 \pm 6.89$ & & $0.17 \pm 2.72$ & $0.38 \pm 2.14$ & $0.58 \pm 2.89$ \\
\hline 20.0 & $17.81 \pm 0.64$ & $18.87 \pm 0.75$ & $17.63 \pm 3.86$ & 0.0 & $0.38 \pm 0.40$ & $0.48 \pm 0.46$ & $0.44 \pm 2.01$ \\
\hline 10.0 & $6.37 \pm 1.43$ & $6.69 \pm 2.01$ & $5.77 \pm 3.89$ & 0.0 & $0.13 \pm 2.61$ & $0.15 \pm 2.14$ & $0.44 \pm 2.41$ \\
\hline
\end{tabular}

It was discussed (Section II) that the accuracy of the PIV displacement vectors is primarily determined by the ratio of the correlation peak size, $d$, to the sub-region size $N$, as shown in Eq. 4 . Nominal values for the peak position error, $\sigma_{\Delta x}$ are on the order of 0.1-pixel for particles spanning 1-2 pixels. However, it was seen in the present experiment that the imaged particles $\left(d_{e}\right)$ span approximately 2-5 pixels depending on which pattern is being imaged. Substituting $d_{e}$ and $N=16$ into Eq. 4 results in a "new" nominal error $\sigma_{\Delta x}$ ranging from 0.17 -pixel -0.44 -pixel, i. e. 3.22- $\mu \mathrm{m}-8.08-\mu \mathrm{m}$. It is evident in Table 1 that standard deviations associated with the detected shifts for both directions fall either within or below the nominal range of error. It is also obvious, that the error for the horizontal shifts is higher than that of the vertical shifts. It is suspected that the higher horizontal error is due to the patterns being manually shifted 5 times in the horizontal direction via the translation stage, consequently introducing manual human positioning errors. The bias error in the mean detected shift values is most likely a result of the positioning errors. The patterns were not manually shifted in the vertical direction and therefore should have less error, as seen in the data; in fact, all of the detected vertical shift error is below the nominal expected range of error. To further investigate the lower range of error associated with the detected shifts, a simulated shift of 36.5- $\mu \mathrm{m}$ (2-pixels) was applied across an image (for both the horizontal and vertical 
directions) via MATLAB and cross-correlated with the corresponding non-shifted image using the PIV software. Introducing a simulated shift eliminates any additional human/experimental error based purely on characteristics of the imaged particles and results in what is believed to be the lower bound of the correlation peak estimation error. A total of 165,668 spatially averaged displacement vectors resulting from the cross-correlation routine for each pattern image pair were averaged to produce an overall background displacement vector, again referred to as the "detected shift". Table 2 shows the detected shifts and their standard deviation for each particle pattern compared to the simulated shift for both the horizontal and vertical directions.

Table 2: Summary of the detected shifts for each particle pattern compared to the simulated shift in both the horizontal and vertical directions.

\begin{tabular}{|c|c|c|c|c|c|c|c|}
\hline $\begin{array}{c}\text { Simulated } \\
\text { Horizontal } \\
\text { Shift } \\
(\boldsymbol{\mu m})\end{array}$ & $\begin{array}{c}\text { Reflective } \\
\text { Adhesive } \\
\text { Detected } \\
\text { Horizontal } \\
\text { Shift } \\
(\boldsymbol{\mu m})\end{array}$ & $\begin{array}{c}\mathbf{4 0 - 1 0 0} \boldsymbol{\mu m} \\
\text { Glass Beads } \\
\text { Detected } \\
\text { Horizontal } \\
\text { Shift } \\
(\boldsymbol{\mu m})\end{array}$ & $\begin{array}{c}\text { Black Paint } \\
\text { Speckles } \\
\text { Detected } \\
\text { Horizontal } \\
\text { Shift } \\
(\boldsymbol{\mu m})\end{array}$ & $\begin{array}{c}\text { Simulated } \\
\text { Vertical } \\
\text { Shift } \\
(\boldsymbol{\mu m})\end{array}$ & $\begin{array}{c}\text { Reflective } \\
\text { Adhesive } \\
\text { Detected } \\
\text { Vertical } \\
\text { Shift } \\
(\boldsymbol{\mu m})\end{array}$ & $\begin{array}{c}\mathbf{4 0 - 1 0 0 \mu m} \\
\text { Glass Beads } \\
\text { Detected } \\
\text { Vertical } \\
\text { Shift } \\
(\boldsymbol{\mu m})\end{array}$ & $\begin{array}{c}\text { Black Paint } \\
\text { Speckles } \\
\text { Detected } \\
\text { Vertical } \\
\text { Shift } \\
(\boldsymbol{\mu m})\end{array}$ \\
\hline 36.54 & $36.54 \pm 0.24$ & $36.51 \pm 0.58$ & $34.54 \pm 4.72$ & 0.0 & $\begin{array}{c}2.17 \mathrm{E}-8 \\
\pm 0.13\end{array}$ & $\begin{array}{c}2.98 \mathrm{E}-8 \\
\pm 0.16\end{array}$ & $\begin{array}{c}4.61 \mathrm{E}-7 \\
\pm 1.11\end{array}$ \\
\hline 0.0 & $\begin{array}{c}1.28 \mathrm{E}-8 \pm \\
0.10\end{array}$ & $\begin{array}{c}3.73 \mathrm{E}-8 \\
\pm 0.38\end{array}$ & $\begin{array}{c}6.30 \mathrm{E}-7 \\
\pm 1.20\end{array}$ & 36.54 & $36.54 \pm 0.16$ & $36.51 \pm 0.67$ & $34.74 \pm 4.61$ \\
\hline
\end{tabular}

It is evident that both the reflective adhesive and the glass beads are more effective at detecting both the manually induced and simulated shifts, compared to the black speckles. For the given range of induced horizontal shifts via the translation mechanism, the black speckles resulted in an average approximate error of 3.5 times more than the error of the glass beads and reflective adhesive. Even more so, the black speckles had an average lower bound error of approximately 24 times the reflective adhesive and 7.5 times the glass beads for the simulated shifts of 36.54-mm (horizontal and vertical). The black speckles also had a bias error of approximately 2 times that of the reflective adhesive and the glass beads for the 50- $\mu \mathrm{m}$ manually induced horizontal shift, which is the expected radial growth of the disk. In addition, it is observed for the cases of the manually induced horizontal shifts of $20-\mu \mathrm{m}$ and $10-\mu \mathrm{m}$ (Table 1) that the black speckle error is less than what was found to be the lower bound error using the simulated shift. Whereas the errors for the reflective adhesive and glass beads for the induced shifts are all above their corresponding lower bound error as expected. The discrepancy for the two cases of black speckle error below the lower bound is noted without clear explanation at this time. However, due to the significantly larger lower bound error of the black speckles and the increased bias error in the range of the expected radial growth of the disk, the black speckled pattern is considered to be a less effective pattern for the disk application as compared to the adhesive and glass beads and therefore will not be considered for future application onto the rotating disk.

When comparing the results of the glass beads to the "ideal" reflective adhesive, the results are considered to be comparable. The adhesive was not considered to be an option for application onto the rotating disk due to its added complexity and uncertainty of the growth of the adhesive versus the growth of the disk. The results of the adhesive were intended to serve a "benchmark" for what can be achieved with uniform particle distribution, since particle density is very difficult to control for the manual applications of particles. Both the glass beads and the adhesive very closely detected the simulated shift. Although the error for the glass beads is 2.4 times that of the reflective adhesive, the average glass bead lower bound error leads to correlation peak estimates that are accurate to $\sim 0.79 \%$ of full scale, which is an optimal range of error; note optimal PIV setup leads to velocity estimates accurate to nominally $1 \%$ of full scale ${ }^{9}$. However, in the case of the 50- $\mu \mathrm{m}$ induced shift, again which is the amount of expected radial growth, the glass bead error in the detected shift is $\sim 6.5 \%$ of full scale, leading to the conclusion that human/experimental error is present; in future experiments, extensive care will be taken in an effort to reduce these errors. Due to the potentially low error capability of the glass beads it is concluded that the glass bead pattern is an effective pattern to be implemented onto the rotating disk in the Rotorydynamics Lab for future testing of the cracked disk. However, before moving forward with the rotating disk application, more work will be performed on the manual application of the beads in an effort to increase the uniformity of the glass beads distribution, since it was shown that a more uniform distribution of particles via the reflective adhesive resulted in lower error.

\section{B. Implementation of the optical technique into the High Precision Rotordynamics Laboratory}


It was concluded from the proof-of-concept experiment that the $40-\mu \mathrm{m}-100-\mu \mathrm{m}$ glass beads will be used as the particle pattern that will be applied on the rotating disk for use in the Rotordynamics Lab. Currently it is proposed to use the 12.7-mm thick, 254-mm diameter aluminum disk (Fig. 8) in which computational analysis has been performed to estimate the amount of expected radial growth for different size notches/induced flaws. Figure 12 compares the computed maximum radial growth of the disk versus rotational speed for the proposed aluminum disk machined with $12.7-\mathrm{mm}, 25.4-\mathrm{mm}$, and $50.8-\mathrm{mm}$ notches. It is shown that the maximum radial displacement will occur for a notch $50.8-\mathrm{mm}$ in length while spinning at $15,000 \mathrm{rpm}$. A contour plot depicting the expected radial displacement is shown in Fig. 13. It is observed that the disk experiences most of the radial growth on the outside edge of the crack and on the outer edge of the disk. It is also observed that disk appears to experience non-uniform growth in the entire cracked vicinity (experiences a strain field), whereas more uniform and lower amounts of growth is observed on the disk opposite of the crack. Note the contour plot has been "exaggerated" in the cracked region of the disk for increased visualization of the strained and deformed areas of the disk immediately surrounding the crack. The black box indicates the expected field-of-view that will be acquired during the rotating disk experiment. The units on the contour scale are micrometers.

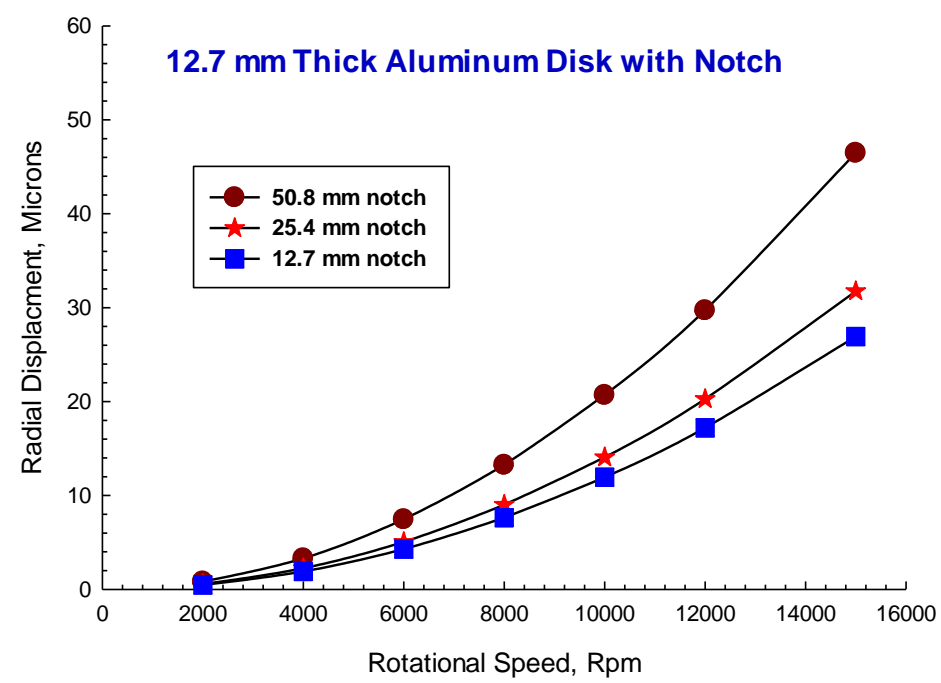

Figure 12. Computed radial displacement vs. rotational speed for the 12.7-mm thick, 254-mm diameter aluminum disk machined with 12.7- $\mathrm{mm}, 25.4-\mathrm{mm}$, and 50.8-mm notches. The largest amount of radial growth occurs for the 50.8-mm notch at 15,000 rpm.
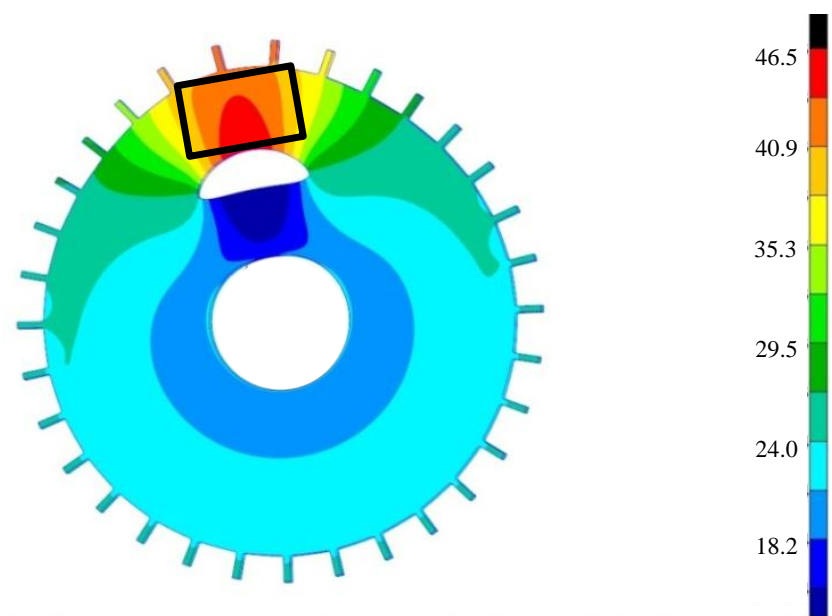

Figure 13. An exaggerated contour diagram depicting the calculated radial deformation of a 12.7-mm, 254-mm diameter disk with a $50.8-\mathrm{mm}$ notch at $15,000 \mathrm{rpm}$ for visualization purposes. Note the black box indicates the field-of-view that will be acquired during the rotating disk experiment and the units on the contour scale are micrometers. 
Future goals involve mapping the strain field experienced by the cracked disk under loaded conditions. Knowing the total deformation (radial growth of the disk) and the original radius of the disk enables calculation of the strain. In order to utilize this technique with a rotating disk it will be necessary to essentially "freeze" the disk when capturing the image of the disk under loaded conditions. It is proposed to "freeze" the disk using a 505-nm LED that will be pulsed on the order of $1-\mu \mathrm{s}$. Fiducial marks will be placed on the disk to facilitate proper registration of the disk reference and data images during post-processing. It should be possible to map out the strain field over the entire circumference of the disk by capturing several images at various points in the disk's rotation cycle. This experiment will be carried out in the near future.

\section{CONCLUSIONS AND FUTURE WORK}

A proof-of-concept experiment was performed in order to develop and validate a non-intrusive, cross-correlation based optical strain measurement technique. The basis of the optical strain measurement technique under investigation consisted of measuring a shift of a high-contrast random particle pattern by imaging the illuminated particles with a CCD camera and using the cross-correlation algorithms in PIV software to evaluate the particle displacements across the area of interest. Three patterns consisting of micro glass beads, retro-reflective adhesive and flat black speckled paint were developed using PIV optimization guidelines: the particle displacement should be less than $1 / 4^{\text {th }}$ of the subregion size, the particle diameter should span approximately 1-2 pixels, and there should be nominally 10 particles per sub-region. In this initial study, three known shifts of 50.0- $\mu \mathrm{m}, 20.0-\mu \mathrm{m}, 10.0-\mu \mathrm{m}$ were induced on each pattern using a translation stage equipped with a fine micrometer. A reference and test image was acquired before and after the induced shift, respectively, and then processed using the PIV software. This procedure was carried out 5 times for each pattern. In addition, an image for each pattern was artificially shifted using software and then cross-correlated with the nonshifted image via the PIV software in order to eliminate any sources of experimental error beyond the cross correlation peak estimation error directly related to the peak correlation algorithm. The measured displacements of the artificially shifted image pair resulted in what is believed to be the lower bound of the correlation peak estimation error for each particle pattern and was used to analyze the effectiveness of each pattern at resolving the induced shifts. Analysis of the results showed that the black speckles had an average lower bound error of approximately 24 times the reflective adhesive and 7.5 times the glass beads for the simulated shifts. The black speckles also had a bias error of approximately 2 times that of the reflective adhesive and the glass beads for the $50-\mu \mathrm{m}$ manually induced horizontal shift, which is the expected radial growth of the disk. The patterns composed of the glass beads and the reflective adhesive were comparable to one another and both more accurately detected the various shifts than the black speckled pattern. Although the glass beads resulted in 2.4 times greater lower bound error than the reflective adhesive, the average glass bead lower bound error resulted in correlation peak estimates that were accurate to $\sim 0.79 \%$ of full scale, which is within the optimal range of error. In the case of the 50- $\mu \mathrm{m}$ manually induced shift, the amount of expected radial growth, the glass bead error in the detected shift was $\sim 3.5 \%$ of full scale, leading to the conclusion that human/experimental error was present and extensive care needs to be taken in an effort to reduce these errors in the future. Due to the high error of the black speckles and the low error capability of the glass beads it was concluded that the glass bead pattern is an effective pattern to be implemented onto the rotating disk in the High Precision Rotordynamics Laboratory at NASA Glenn Research Center.

Prior to implementation of the glass bead pattern onto the disk, future work will be conducted in an effort to further reduce the effective particle diameter in order to obtain the optimal 1-2 pixel diameter of the imaged particle. Currently the effective particle diameter of the glass beads is 2-3 pixels. Having the particles span the optimal imaged particle diameter may further reduce the error in the radial displacement estimates. This work will include investigating alternative light sources, which may facilitate the use of a smaller f/\# setting and thereby decrease the diffraction limited blur circle, thus decreasing the effective particle diameter. Furthermore, increased time will be spent investigating the availability and options of smaller glass beads, which will also decrease the effective particle diameter, which may enable the achievement of the optimal imaged particle diameters. In addition, more work will be performed on the manual application of the beads in an effort to increase the uniformity of the glass beads distribution, since it was shown that a more uniform distribution of particles via the reflective adhesive resulted in lower error. The refined glass bead pattern will be applied to the disk in an effort to detect radial displacements of the disk due to a localized strain field surrounding an induced crack under rotation at $15,000 \mathrm{rpm}$. These investigations are being carried out for the development optical surface measurements for the internal parts of a flow, such as the engine turbine disk, and are supported by the Innovative Measurements Task within the Aeronautical Sciences Project under NASA's Fundamental Aeronautics Program. 


\section{ACKNOWLEDGEMENTS}

This work was supported by the Aeronautical Sciences Project under NASA's Fundamental Aeronautics Program. In addition, the author would like to acknowledge Dr. Mark Wernet for use of his PIV software package.

\section{REFERENCES}

[1] Woike, M., Abdul-Aziz, A., Bencic, T., "A Microwave Blade Tip Clearance Sensor for Propulsion Health Monitoring,” NASA/TM-2010-216736, AIAA-2010-3308, 2010.

[2] Woike, M., Abdul-Aziz, "Crack Detection Experiments on Simulated Engine Turbine Disks at NASA Glenn Research Center's Rotordynamics Laboratory,” NASA/TM-2010-216239, AIAA-2010-587, 2010.

[3] Woike, M., Roeder, J., Hughes, C., and Bencic, T., "Testing of a Microwave Blade Tip Clearance Sensor at the NASA Glenn Research Center," NASA/TM-2009-215589, AIAA-2009-1452, 2009.

[4] Abdul-Aziz, A., Woike, M., Oza, N., Matthews, B., and Baaklini G., "Propulsion Health Monitoring of a Turbine Engine Disk Using Spin Test Data,” NASA/TM—2010-216743, 2010.

[5] Woike, M., Abdul-Aziz, A., Fralick, G., and Wrbanek, J., "Investigaton of a Moiré Based Crack Detection Technique for Propulsion Health Monitoring," NASA/TM - 2012-217622, SPIE, 2012.

[6] Wernet, M., "Fuzzy Logic Enhanced Digital PIV Processing Software," NASA/TM-1999-209274, 1999.

[7] Wernet, M., Pline, A., "Particle Placement Tracking Technique and Cramer Rao Lower Bound Error in Centroid Estimates in from CCD Imagery," Experiments in Fluids, Vol. 15, No. 4-5, pp.295-307, 1993.

[8] Adrian, R. J., "Multi-Point Optical Measurements of Simultaneous Vectors in Unsteady Flow-A Review," Int. J. of Heat and Fluid Flow, Vol. 7, pp. 127-145, 1986.

[9] Westerweel, J., "Fundamentals of Digital Particle Image Velocimetry," Meas. Sci. Technol., Vol. 8, pp. 1379-1392, 1997.

[10] Westerweel, J., "Theoretical analysis of the measurement precision in particle image velocimetry," Experiments in Fluids, [Suppl], pp. S3-S12, 2000.

[11] Keane, R., Adrian, R., "Theory of cross-correlation analysis of PIV images," Applied Scientific Research, Vol. 49, pp.191-215, 1992.

[12] Mercer, C., [Optical Metrology for Fluids, Combustion, and Solids], Kluwer Academic Publishers, Boston, pp. 69$104,(2003)$.

[13] Raffel, M., Willert, C., Kompenhans, J., [Particle Image Velocimetry], Springer, Verlag Berlin Heidelberg New York, (1998). 